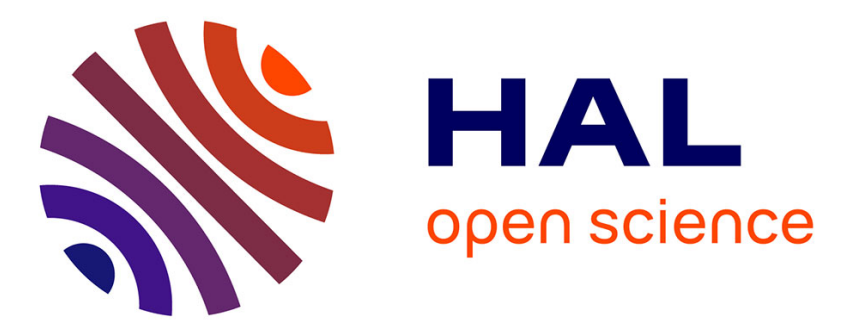

\title{
Coherent Control of Ballistic Photocurrents in Multilayer Epitaxial Graphene Using Quantum Interference
}

\author{
Dong Sun, Charles Divin, Julien Rioux, J. E. Sipe, Claire Berger, Walt A. de
} Heer, Philip N. First, Theodore B. Norris

\section{To cite this version:}

Dong Sun, Charles Divin, Julien Rioux, J. E. Sipe, Claire Berger, et al.. Coherent Control of Ballistic Photocurrents in Multilayer Epitaxial Graphene Using Quantum Interference. Nano Letters, 2010, 10, pp.1293-1296. 10.1021/nl9040737 . hal-01002970

\section{HAL Id: hal-01002970 https://hal.science/hal-01002970}

Submitted on 7 Jun 2014

HAL is a multi-disciplinary open access archive for the deposit and dissemination of scientific research documents, whether they are published or not. The documents may come from teaching and research institutions in France or abroad, or from public or private research centers.
L'archive ouverte pluridisciplinaire HAL, est destinée au dépôt et à la diffusion de documents scientifiques de niveau recherche, publiés ou non, émanant des établissements d'enseignement et de recherche français ou étrangers, des laboratoires publics ou privés. 


\title{
Coherent Control of Ballistic Photocurrents in Multilayer Epitaxial Graphene Using Quantum Interference
}

\author{
Dong Sun,$\uparrow$ Charles Divin,$\uparrow$ Julien Rioux, $₫$ John E. Sipe, $\$$ Claire Berger, $\S$ Walt A. de Heer, $\S$ \\ Phillip N. First, $\S$ and Theodore B. Norris*,$\dagger$ \\ †Center for Ultrafast Optical Science, University of Michigan, Ann Arbor, Michigan 48109- \\ 2099, \\ $\neq$ Department of Physics and Institute for Optical Sciences, University of Toronto, Ontario, \\ Canada M5S 1A7, \\ $\S$ School of Physics, Georgia Institute of Technology, Atlanta, Georgia 30332
}

\begin{abstract}
We report generation of ballistic electric currents in unbiased epitaxial graphene at $300 \mathrm{~K}$ via quantum interference between phase-controlled cross-polarized fundamental and second harmonic $220 \mathrm{fs}$ pulses. The transient currents are detected via the emitted terahertz radiation. Because of graphene's special structure symmetry, the injected current direction can be well controlled by the polarization of the pump beam in epitaxial graphene. This all optical injection of current provides not only a noncontact way of injecting directional current in graphene but also new insight into optical and transport process in epitaxial graphene.
\end{abstract}


Proposals for graphene-based spintronics, ${ }^{1}$ valleytronics, ${ }^{2}$ and quantum computing ${ }^{3}$ attest to the intense interest in graphene for future electronic device applications. ${ }^{4-9}$ The ability of graphene to support ballistic electron transport ${ }^{10-13}$ is central to many applications, and thus a major problem is the injection and detection of ballistic currents. One solution from the field of optoelectronics is the use of coherent control of quantum interference between different absorption pathways. In this report, we demonstrate a coherent control strategy for launching ballistic current pulses in arbitrary directions within a graphene layer.

The ability to optically inject currents in specified directions has been demonstrated in a wide variety of atomic gases ${ }^{14-17}$ and semiconductor materials. ${ }^{18-22}$ In a typical experiment, two phase-locked beams at a fundamental $(\boldsymbol{\omega})$ and second-harmonic $(2 \boldsymbol{\omega})$ frequency are incident on a sample. In a semiconductor, the $2 \omega$ beam generates electron hole pairs via one-photon interband transitions; the $\omega$ beam generates electron-hole pairs via two-photon transitions. The one- and two-photon absorption pathways can interfere, leading to a phase-dependent directional ballistic electron current injection, with constructive (destructive) interference at $\mathrm{k}$ $(-\mathrm{k})$, or vice versa.

The possibility of producing and detecting coherently controlled photocurrents in single layer graphene, although a controversial suggestion, ${ }^{22,23}$ is exciting for at least two reasons. First, the technique would provide a noncontact, all-optical way to inject directional current, eliminating parasitic effects due to electrical contacts for fundamental studies and possible applications; indeed, calculations show that the direction of the injected current can be well controlled by the relative polarization of the pump beams. Second, a quantitative study of the fate of the injected current could provide new insight into electronic scattering processes in graphene emitted by ballistic photocurrents generated via quantum interference between phase-controlled, cross-polarized fundamental and second harmonic 220 fs pulses in unbiased multilayer epitaxial graphene (MEG) at $300 \mathrm{~K}$.

For any crystalline material, a Fermi's Golden Rule calculation ${ }^{18}$ predicts a current density injection rate of the form $\mathbf{J}=\boldsymbol{\eta}_{\boldsymbol{\omega}}: \mathbf{E}_{-\boldsymbol{\omega}} \mathbf{E}_{-\boldsymbol{\omega}} \mathbf{E}_{2 \boldsymbol{\omega}}+\mathbf{c . c}$., where $\boldsymbol{\eta}_{\boldsymbol{\omega}}$ is a fourth-rank tensor and $\mathbf{E}_{\boldsymbol{\omega}}$ and $\mathbf{E}_{2 \omega}$ are the (complex) field amplitudes, with phases $\varphi_{\omega}$ and $\varphi_{2 \omega}$ respectively. Using a tight-binding model with isotropic bands, ${ }^{6}$ for a single graphene sheet subject to linearly polarized light at normal incidence the current injection is controlled by one independent parameter of the tensor $\boldsymbol{\eta}_{\boldsymbol{\omega}}$, given by $\boldsymbol{\eta}_{\boldsymbol{\omega}}=\mathbf{g}_{\mathrm{s}} \mathbf{g}_{\mathrm{v}} \mathrm{e}^{4} \mathbf{v}_{\mathrm{F}} 2(2 \boldsymbol{h} \boldsymbol{\omega})^{-3}$, where $\mathbf{g}_{\mathrm{s}, \mathrm{v}}$ are the spin and valley degeneracies, $\mathrm{e}$ is the electron charge, and $\mathbf{v}_{\mathrm{F}}$ the Fermi velocity. The current density injection takes the form

$\dot{j}=2 \eta_{\omega}\left|E_{\omega}\right|^{2}\left|E_{2 \omega}\right| \sin \left(2 \phi_{\omega}-\phi_{2 \omega}\right) \hat{n}$

where the direction $\hat{n}=\hat{e}_{2 \omega} \cos 2 \theta+\hat{\mathrm{e}}_{2 \omega}^{\frac{1}{2 \omega}} \sin 2 \theta$ of the injected current depends on the angle $\theta$ between the polarization of the fundamental and second harmonic beams and is independent of the orientation of the graphene crystal axes; here $\mathbf{e}_{2 \omega}{ }_{\text {identifies the }}$ polarization of the second harmonic and $\mathbf{e}^{\wedge}{ }_{2 \omega}$ is a vector in the graphene plane perpendicular to $\mathbf{e}^{\hat{2} \omega}$. Thus even in MEG samples, where the orientation of the crystal axes can vary from 
one graphene layer to the next, the injected ballistic current can be controlled in both magnitude and direction by adjusting the beam parameters. The coherent injection of ballistic carriers with ultrashort pump pulses leads to a current surge that is damped by a combination of scattering (momentum relaxation) and the build-up of space charge. This transient current is directly observed via the emitted terahertz radiation. ${ }^{19,22}$

The specific experimental conditions are as follows. A 250-kHz Ti:sapphire oscillator/amplifier (Coherent RegA) operating at $800 \mathrm{~nm}$ is used to pump an optical parametric amplifier (OPA), followed by a differential frequency generator (DFG) to generate $2-4 \mathrm{~mW}$ of 3.2 or $4.8 \mu \mathrm{m}(\boldsymbol{\omega}$ beam) with $220 \mathrm{fs}$ pulse width. The $\boldsymbol{\omega}$ beam passes through an $\mathrm{AgGeS} 2$ (for $3.2 \mu \mathrm{m}$; conversion efficiency $(\mathrm{CE}) \approx 10 \%$ ) or $\mathrm{ZnGeP} 2$ (for $4.8 \mu \mathrm{m}$; CE g 10\%) crystal (type I) to generate cross-linearly polarized pulses at 1.6 or $2.4 \mu \mathrm{m}$ ( $2 \omega$ beam). The collinearly propagating $\boldsymbol{\omega}$ and $2 \boldsymbol{\omega}$ pulses pass through a CaF2 plate with tunable tilt angle to adjust the relative phase. The two emerging pump beams are overlapped on the sample with a $15 \mu \mathrm{m}$ diameter (fwhm) spot size, producing peak focused intensities on the sample of 2.8 $\mathrm{GW} / \mathrm{cm}-2$ (at $\lambda=3.2 \mu \mathrm{m}$ ) and $0.45 \mathrm{GW} / \mathrm{cm}-2$ (at $\lambda=1.6 \mu \mathrm{m}$ ) after losses due to intermediate optics. The coherently radiated terahertz pulse generated by the ballistic electrons is detected in the far field using electro-optic sampling in a $1 \mathrm{mmt}$ hick (110)- oriented ZnTe crystal; a polyethylene plate transparent to terahertz radiation is used to block the transmitted mid-IR beam. The effective bandwidth of the electro-optic detection system is estimated to be $\sim 2$ $\mathrm{THz}$, limited by phase mismatch between the terahertz and $800 \mathrm{~nm}$ sampling beams.

The sample is a multilayer epitaxial graphene film produced on the C-terminated face of single-crystal $4 \mathrm{H}-\mathrm{SiC}$ by thermal desorption of $\mathrm{Si}^{10}{ }^{10}$ Samples used have from 9 to 63 graphene atomic layers; such samples have been shown to have the graphene linear band structure and are distinct from graphite. ${ }^{13,24-28}$ The first few layers are heavily doped with the doping decreasing to zero gradually. The Fermi levels of the first four doped layers are measured in a separate experiment to be 365, 220, 140, and $93 \mathrm{meV}$ above the Dirac point respectively. ${ }^{29}$ As shown in Figure 1, the $1.6 \mu \mathrm{m}$ wavelength corresponding to the $2 \omega$ beam is chosen to excite electrons immediately above the Fermi level of the most heavily doped layer. One-photon absorption of the $\boldsymbol{\omega}$ beam is not allowed in this doped layer due to Pauli blocking; however, this process would inject electrons with no net average velocity in the undoped (or slightly doped) layers of the sample. Such electrons are not injected in experiments on semiconductors when the $\boldsymbol{\omega}$ beam lies within a bandgap; they are unavoidable in the graphene sample, and we refer to them as "bad electrons," since they may degrade the ballistic photocurrent.

Figure 2 a shows the measured terahertz field as a function of time delay between the 3.2 $\boldsymbol{\mu m} / 1.6 \mu \mathrm{m}$ pump pulses and an electro-optic sampling $800 \mathrm{~nm}$ probe pulse; traces are shown for two values of the phase difference $\Delta \Phi=2 \Phi_{\omega}-\Phi_{2 \omega}$ separated by $\pi$. The oscillatory temporal waveform is a result of the finite bandwidth of the electro-optic detection system rather than the dynamics of the system. As shown in Figure 2a, the terahertz field reverses sign when $\Delta \Phi$ changes by $\boldsymbol{\pi}$, which is consistent with a coherence-induced current source. The current amplitude and direction can be controlled through the phase parameter $\Delta \Phi$ alone. Figure $2 \mathrm{~b}$ shows a contour plot of the terahertz radiation field from MEG as a function of $\Delta \Phi$ and the time delay between the $3.2 \mu \mathrm{m} / 1.6 \mu \mathrm{m}$ pump pulses and $800 \mathrm{~nm}$ probe pulse. A typical terahertz pulse trace as a function of the sampling pulse time delay at constant $\Delta \Phi$ is shown in 
the top panel and corresponds to the horizontal dashed line on the contour plot. The right panel shows the dependence of the terahertz field on $\Delta \Phi$ at constant sampling pulse delay. The current reverses direction as the phase varies and more generally follows a $\sin (\Delta \Phi)$ dependence, consistent with the coherently controlled photocurrent description of eq 1 . To further verify that the terahertz radiation signal is associated with the two color current injection process, we measured the scaling of current generation with the pump power of $\boldsymbol{\omega}$ beam before the second harmonic generation crystal. From eq 1, we expect $\left|\mathbf{E}_{\mathrm{THz}}\right| \boldsymbol{\alpha}|\mathbf{J}| \boldsymbol{\alpha}$ $\mathbf{P}_{\boldsymbol{\omega}}\left(\mathbf{P}_{2 \boldsymbol{\omega}}\right)^{1 / 2} \boldsymbol{\alpha}\left(\mathbf{P}_{\boldsymbol{\omega}}\right)^{2}$. The experiment data in Figure 2c supports the expected power dependence and is consistent with a third order optical process.

We next consider polarization effects on the direction of the coherent photocurrent. For linear polarizations, the predictions are given by eq 1 , and for cocircularly polarized pump beams a similar calculation predicts that the phase parameter $2 \boldsymbol{\Phi}_{\omega}-\boldsymbol{\Phi}_{2 \omega}$ controls the direction of the current, again with a direction insensitive to the crystal orientation of the graphene. Since the $\omega$ and $2 \omega$ beams copropagate in the experiment, the polarization of the pump beams cannot be adjusted separately to fully test these predictions. Instead, we use a half-wave plate to rotate the $\omega$ beam polarization, and at the same time rotate the second harmonic crystal following the half wave plate to optimize the second harmonic generation; this maintains cross-linear polarization of the pump beams. From eq 1, the photocurrent direction and thus the terahertz polarization should follow the polarization of the $2 \omega$ beam. To test this we put a terahertz polarizer after the sample; after projecting on a fixed terahertz polarizer, the transmitted terahertz field should follow a sinusoidal curve. This is confirmed by the experimental data shown in Figure 3.

Further experiments were performed using 4.8 um and 2.4 um pump wavelengths, and on samples with different numbers of graphene layers. Figure 4 shows the peak terahertz amplitude at different positions on four different samples with either $3.2 \mu \mathrm{m} / 1.6 \mu \mathrm{m}$ or 4.8 $\mu \mathrm{m} / 2.4 \mu \mathrm{m}$ pump wavelengths, as a function of the number of graphene layers. Even with the presence of some sample inhomogeneity, it is clear that the signal amplitude does not increase monotonically with increasing number of graphene layers. This is simply because each graphene layer absorbs both terahertz radiation and the mid-IR pump beams. To model this, we assume a flat spectrum with an absorption coefficient of $2.3 \%$ at all wavelengths, 30 appropriate for one-photon absorption. The emitted terahertz radiation should be proportional to $\left|\mathbf{E}_{\mathrm{THz}}\right| \boldsymbol{\alpha}\left|\mathbf{E}_{\omega}{ }^{2} \mathbf{E}_{2 \omega}\right|$ from eq 1, so the enhancement factor of $\mathbf{N}$ layers with respect to a single layer is

$$
E_{n}=\sum_{k=1}^{N}\left(x^{k-1}\right)^{3} x^{N-k}
$$

where $\mathbf{x}=(1-2.3 \%)^{1 / 2}$ is the transmission coefficient of electric field by one graphene layer. This fitting curve predicts an optimum layer number of 47 , which is in good agreement with experiment, as shown in Figure 4. Now the frequency independence of the linear absorption coefficient ${ }^{30}$ corresponds to a one-photon carrier injection rate proportional to $\omega^{-1}$; the corresponding two-photon absorption calculation predicts a carrier inject rate proportional to $\omega^{-5}$. This could indicate a different importance of the "bad" electrons at different excitation frequencies. But since we can fit the trend of the experimental data in Figure 4 by assuming an equal contribution of the signal from each graphene layer, apparently the "bad electrons" excited in the undoped layers do not significantly degrade the coherent current. We can also 
rule out the possibility that the highly doped layer dominates the contribution to the terahertz signal.

In conclusion, we have injected coherently controlled electrical currents in MEG using phasecontrolled 220 fs pulse pairs at $3.2 \mu \mathrm{m} / 1.6 \mu \mathrm{m}$ and $4.8 \mu \mathrm{m} / 1.6 \mu \mathrm{m}$. The ballistic current amplitude is determined by the relative phase between the pulses. The injected current direction can be fully controlled by the polarization of the pump beams and does not rely on sample orientation. The theoretically predicted current direction variation with linear polarization excitation has been verified experimentally. This opens the door to further experimental work to verify the purely phase controlled current direction under circularly polarized excitation and to exploit this effect in novel ballistic electron devices.

\section{Referenes and Notes}

(1) Tombros, N.; Jozsa, C.; Popinciuc, M.; Jonkman, H. T.; van Wees, B. J. Electronic spin transport and spin precession in single graphene layers at room temperature. Nature 2007, 448, 571- 574.

(2) Rycerz, A.; Tworzydlo, J.; Beenakker, C. W. J. Valley filter and valley valve in graphene. Nat. Phys. 2007, 3, 172-175.

(3) Trauzettel, B.; Bulaev, D. V.; Loss, D.; Burkard, G. Spin qubits in graphene quantum dots. Nat. Phys. 2007, 3, 192-196.

(4) Berger, C.; et al. Ultrathin Epitaxial Graphite: 2D Electron Gas Properties and a Route toward Graphene-based Nanoelectronics. J. Phys. Chem. B 2004, 108, 19912-19916.

(5) De Heer, W. A.; et al. Epitaxial graphene. Solid State Commun. 2007, 143, 92-100.

(6) Geim, A. K.; Novoselov, K. S. The rise of graphene. Nat. Mater. 2007, 6, 183-191.

(7) Novoselov, K. S.; et al. Electric Field Effect in Atomically Thin Carbon Films. Science 2004, 306, 666-669.

(8) Wang, F.; et al. Gate-Variable Optical Transitions in Graphene. Science 2008, 320, 206209.

(9) Zhang, Y.; et al. Direct observation of a widely tunable bandgap in bilayer graphene. Nature 2009, 459, 820-823.

(10) Berger, C.; et al. Electronic Confinement and Coherence in Patterned Epitaxial Graphene. Science 2006, 312, 1191-1196.

(11) Bolotin, K. I.; Sikes, K. J.; Hone, J.; Stormer, H. L.; Kim, P. Temperature-Dependent Transport in Suspended Graphene. Phys. Rev. Lett. 2008, 101, 096802.

(12) Morozov, S. V.; et al. Giant Intrinsic Carrier Mobilities in Graphene and Its Bilayer. Phys. Rev. Lett. 2008, 100, 016602.

(13) Orlita, M.; et al. Approaching the Dirac Point in High-Mobility Multilayer Epitaxial Graphene. Phys. Rev. Lett. 2008, 101, 267601.

(14) Chen, C.; Yin, Y.-Y.; Elliott, D. S. Interference between optical transitions. Phys. Rev. Lett. 1990, 64, 507.

(15) Gurevich, G. L.; Khronopulo, Y. G. Measurements of optical phase variations using interfering multiphoton ionization processes. Sov. Phys. - JEPT 1967, 24, 1012.

(16) Jackson, D. J.; Wynne, J. J.; Kes, P. H. Resonance-enhanced multiphoton ionization: Interference effects due to harmonic generation. Phys. Rev. A 1983, $28,781$. 
(17) Yin, Y.-Y.; Chen, C.; Elliott, D. S.; Smith, A. V. Asymmetric photoelectron angular distributions from interfering photoionization processes. Phys. Rev. Lett. 1992, 69, 2353.

(18) Atanasov, R.; Hach, A.; Hughes, J. L. P.; van Driel, H. M.; Sipe, J. E. Coherent Control of Photocurrent Generation in Bulk Semiconductors. Phys. Rev. Lett. 1996, 76, 1703.

(19) Costa, L.; Betz, M.; Spasenovic, M.; Bristow, A. D.; van Driel, H. M. All-optical injection of ballistic electrical currents in unbiased silicon. Nat Phys 2007, 3, 632-635.

(20) Dupont, E.; Corkum, P. B.; Liu, H. C.; Buchanan, M.; Wasilewski, Z. R. PhaseControlled Currents in Semiconductors. Phys. Rev. Lett. 1995, 74, 3596.

(21) Hach, A.; et al. Observation of Coherently Controlled Photocurrent in Unbiased, Bulk GaAs. Phys. Rev. Lett. 1997, 78, 306.

(22) Newson, R. W.; Menard, J.-M.; Sames, C.; Betz, M.; Driel, H. M. v. Coherently Controlled Ballistic Charge Currents Injected in Single-Walled Carbon Nanotubes and Graphite. Nano Lett. 2008, 8, 1586-1589.

(23) Mele, E. J.; Kral, P.; Tomanek, D. Coherent control of photocurrents in graphene and carbon nanotubes. Phys. Rev. B 2000, 61, 7669.

(24) Faugeras, C.; et al. Few-layer graphene on $\mathrm{SiC}$, pyrolitic graphite, and graphene: A Raman scattering study. Appl. Phys. Lett. 2008, 92, 011914.

(25) Hass, J.; et al. Why Multilayer Graphene on 4H-SiC(000-1) Behaves Like a Single Sheet of Graphene. Phys. Rev. Lett. 2008, 100, 125504.

(26) Miller, D. L.; et al. Observing the Quantization of Zero Mass Carriers in Graphene. Science 2009, 324, 924-927.

(27) Sadowski, M. L.; Martinez, G.; Potemski, M.; Berger, C.; De Heer, W. A. Landau Level Spectroscopy of Ultrathin Graphite Layers. Phys. Rev. Lett. 2006, 97, 266405.

(28) Wu, X.; Li, X.; Song, Z.; Berger, C.; De Heer, W. A. Weak Antilocalization in Epitaxial Graphene: Evidence for Chiral Electrons. Phys. Rev. Lett. 2007, 98, 136801.

(29) Sun, D. et al. Spectroscopy Measurement of Interlayer Screening in Multilayer Epitaxial Graphene. Phys. Rev. Lett. 2010; in press.

(30) Nair, R. R.; et al. Fine Structure Constant Defines Visual Transparency of Graphene. Science 2008, 320, 1308. 


\section{Figure Captions}

FIGURE 1. Schematic energy-momentum band diagram of doped and undoped layers of MEG near the Dirac point. Red is associated with the $\omega$ beam, blue with the $2 \omega$ beam. Asymmetric electron populations at ( $\mathrm{k}$ and hence current generation, is indicated by shaded patches. The dash line indicates the Fermi level of doped and undoped graphene layers.

FIGURE 2. Phase-controlled terahertz emission from injected photocurrent. (a) Timedependent electro-optic signals of terahertz fields from a MEG sample with 63 layers for different values of $\Delta \Phi$. (b) Time-dependent terahertz radiation field from MEG as a function of time delay between $3.2 \mu \mathrm{m} / 1.6 \mu \mathrm{m}$ pump and $800 \mathrm{~nm}$ probe pulse and the phase parameter, $\Delta \Phi$, between cross-polarized pump beams. Top panel: time-domain terahertz signal for the constant $\Delta \Phi$ represented by the horizontal dashed line on the contour plot. Right panel: terahertz field $\Delta \Phi$ dependence for constant time delay represented by the vertical dashed line on the contour plot. (c) Fundamental beam power dependence. The green line gives the dependence of the second harmonic power with respect to the fundamental beam power and the blue line gives the dependence of the generated terahertz peak field on the fundamental beam power. Log-log plots are used to display the power dependence. The fundamental beam wavelength is $4.8 \mu \mathrm{m}$ in this experiment. 


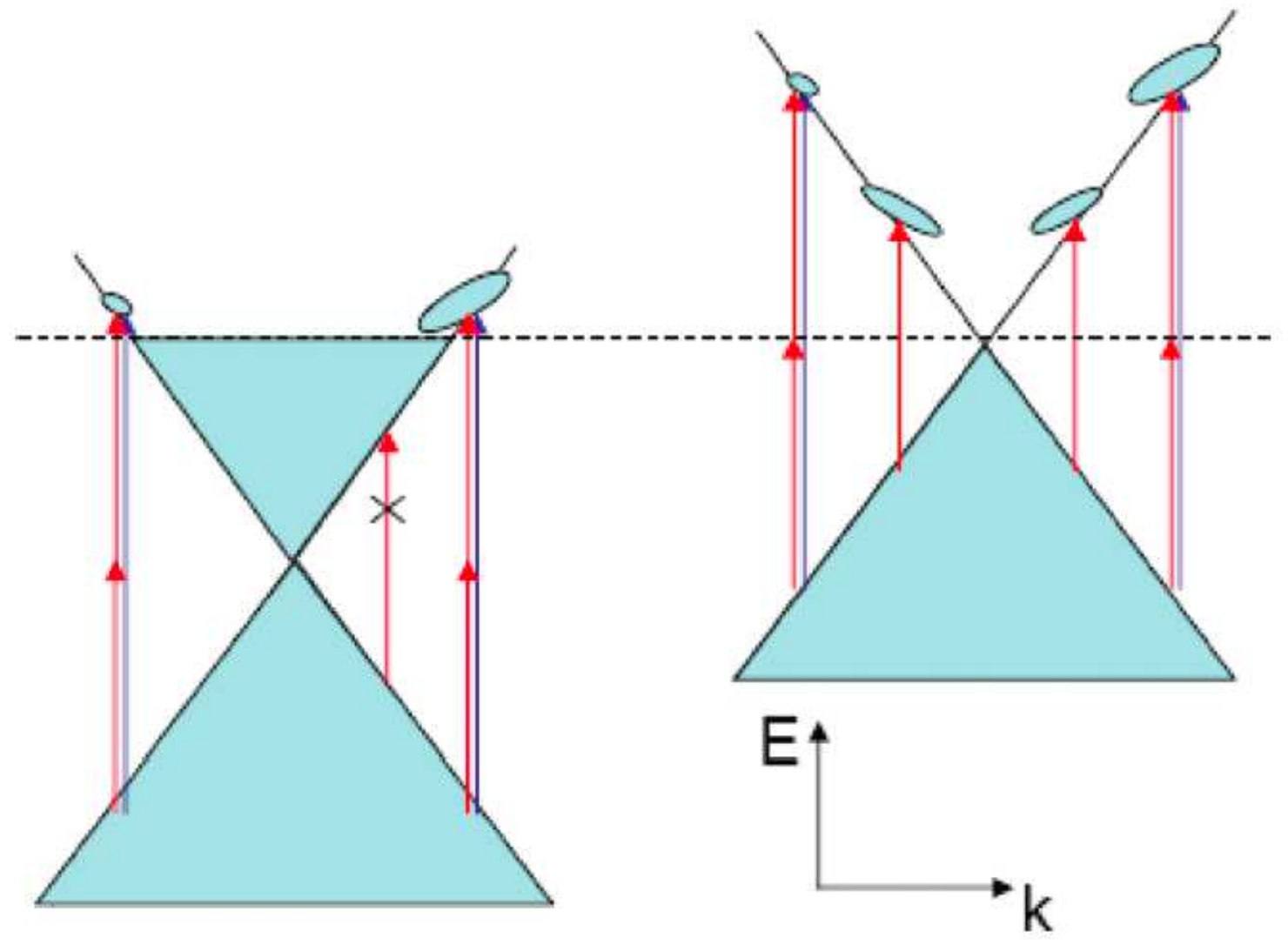



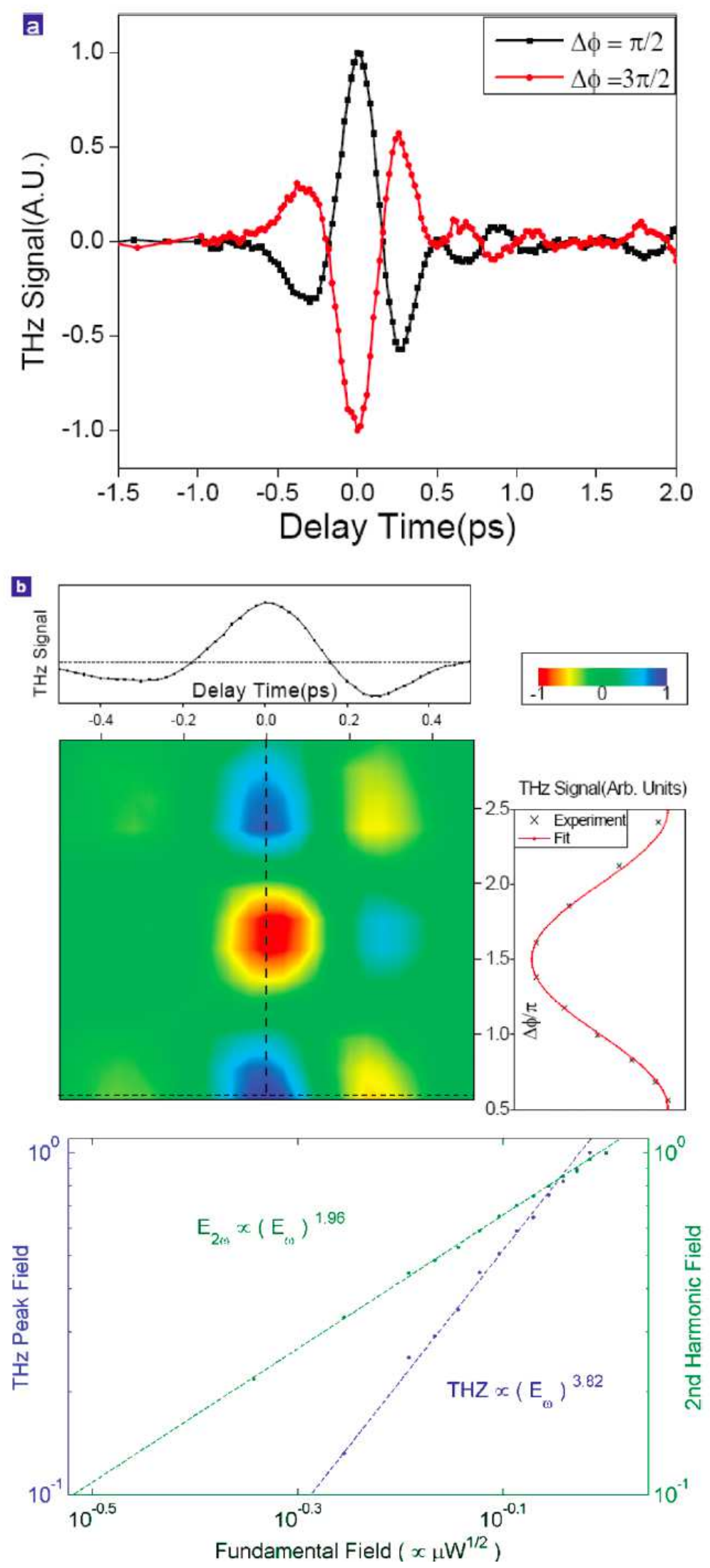
